\title{
Pensamento Computacional: transformando ideias em jogos digitais usando o Scratch
}

\author{
Carla Lopes Rodriguez ${ }^{1}$, Aparecida M. Zem-Lopes ${ }^{1,2}$, Leonardo Marques ${ }^{1}$, \\ Seiji Isotani ${ }^{1}$ \\ ${ }^{1}$ Instituto de Ciências Matemáticas e Computação - Universidade de São Paulo \\ Av. Trabalhador São Carlense, 400- 13566-590 - São Carlos - SP - Brasil \\ ${ }^{2}$ Faculdade de Tecnologia de Jahu - Fatec Jahu \\ Rua Frei Galvão, s/n.o, Jd. Pedro Ometto - Jaú - SP \\ \{carlalrodriguez, cida.zem, leobmarques\} @gmail.com.br, \\ sisotanieicmc.usp.br
}

\begin{abstract}
The integration of Information and Communication Technology in schools routine is required, since they have the potential to transform teaching and learning process and can contribute to the development of various skills in young students, for example, logical reasoning and problem-solving ability. Thus, this work presents the experience in a project carried out under a PreScientific Initiation program, whose main objective was to develop basics principles of "computational thinking" along the first year of high school' students from a public school by using the Scratch features.
\end{abstract}

Resumo. A integração das Tecnologias de Informação e Comunicação à rotina das escolas se faz hoje necessária, uma vez que possuem potencial para transformar o processo de ensino e aprendizagem e podem contribuir para o desenvolvimento de diversas habilidades nos jovens estudantes como, por exemplo, o raciocínio lógico e a capacidade de resolução de problemas. Nesse sentido, esse trabalho apresenta a experiência vivenciada em um projeto, realizado no âmbito de um programa de Pré-Iniciação Científica, cujo objetivo principal foi desenvolver noções básicas do "pensamento computacional", junto aos alunos do primeiro ano do ensino médio de uma escola pública, por meio dos recursos do Scratch.

\section{Introdução}

A informação e a comunicação representam papéis relevantes no processo de transformação política, econômica e social vivido pela sociedade atual. Nesse sentido, novas orientações quanto aos currículos e programas escolares são necessárias para que a educação formal possa contribuir para o desenvolvimento das habilidades cognitivas, sociais e profissionais exigidas (GATTI, 2000). Essas orientações envolvem, por exemplo, a integração das Tecnologias de Informação e Comunicação (TIC), e toda a mudança trazida por estas à rotina escolar. É o que postula o documento Sociedade da Informação no Brasil (MCT, 2000), quando evidencia a necessidade de se adotar metodologias mais adequadas para a integração das TIC na escola, considerando que:

A educação é o elemento-chave na construção de uma sociedade baseada na informação, no conhecimento e no aprendizado (...) educar em uma sociedade da informação significa muito mais que treinar as 
pessoas para o uso das tecnologias de informação e comunicação (...). Trata-se também de formar os indivíduos para "aprender a aprender", de modo a serem capazes de lidar positivamente com a contínua e acelerada transformação da base tecnológica (MCT, 2000, p. 45).

Segundo Costa et al. (2012), reconhecer o potencial transformador que as TIC podem trazer ao processo de ensinar e aprender é o primeiro passo para que seja possível reorganizar currículos e programas escolares de forma que estes apoiem o desenvolvimento das habilidades cognitivas, sociais e profissionais desejadas (VALENTE, 2003). Dentre essas habilidades, o raciocínio lógico e a resolução de problemas são recursos cognitivos importantes para várias áreas do conhecimento (ZAHARIJA et al., 2013; NUNES, 2011).

Raciocinar logicamente para resolver problemas é uma habilidade desenvolvida pelos profissionais da área da computação. Como a programação é fundamental para operar os computadores, a tendência é que esses profissionais pensem nos problemas de forma analítica e desenvolvam soluções em forma de algoritmos (EASTERBROOK, 2014). Essa forma estruturada de pensar foi denominada de "pensamento computacional" por Jeannette Wing e, segundo Lee (2014), é um processo de raciocínio lógico que inclui características como: i) formulação de problemas; ii) organização e análise lógica dos dados; iii) representação por meio de abstrações; iv) soluções automatizadas por meio de algoritmos; v) identificação, análise e implementação de soluções; e vi) generalização e transferência do processo de solução encontrado para resolução de outros problemas. Segundo Wing (2006), o "pensamento computacional" pode representar a contribuição mais importante da ciência da computação para o mundo e deve ser ensinado aos estudantes nas mais diversas disciplinas.

Diante do que foi exposto, o desafio de favorecer o desenvolvimento de habilidades cognitivas, e levar o "pensamento computacional" aos alunos do ensino fundamental e médio, passa pela necessidade de sugerir ferramentas que possam apoiar a aprendizagem de conceitos de lógica de programação de uma forma mais lúdica e adequada a essa faixa etária. Assim, esse trabalho apresenta os procedimentos metodológicos adotados, as atividades desenvolvidas e os resultados alcançados em um projeto realizado no âmbito do programa de Pré-Iniciação Científica (Pré-IC) ${ }^{1}$, para os anos letivos de 2014 (segundo semestre) e 2015 (primeiro semestre). O projeto teve como principal objetivo apresentar e trabalhar noções básicas do "pensamento computacional", junto de sete alunos do primeiro ano do ensino médio de uma escola pública, por meio de tecnologias digitais, no caso, o $\mathrm{Scratch}^{2}$.

Além desta seção introdutória, o artigo está estruturado da seguinte forma: na seção 2 faz-se uma breve apresentação do ambiente de programação Scratch e os conceitos que dele derivam; na seção 3 são apresentados os trabalhos relacionados ao projeto em questão; na seção 4 a metodologia utilizada é descrita; na seção 5 o perfil dos alunos participantes é apresentado; na seção 6 são detalhadas as atividades

\footnotetext{
${ }^{1}$ O programa de Pré-Iniciação Científica (Pré-IC) é uma iniciativa da Pró-Reitoria de Pesquisa da USP, que visa apoiar o desenvolvimento de projetos nas áreas das ciências exatas, biológicas e humanas. O objetivo é despertar e incentivar o interesse de alunos da rede pública de ensino para a ciência, por meio da convivência com os procedimentos e as metodologias da pesquisa científica.

2 Scratch - https://scratch.mit.edu/
} 
desenvolvidas; na seção 7 apresentam-se os principais resultados alcançados com o projeto; e, por fim, na seção 8, são feitas as considerações finais sobre o trabalho.

\section{Scratch}

O Scratch é uma linguagem de programação disponível online, desenvolvida com o objetivo de possibilitar que iniciantes possam criar programas de computador sem aprender a sintaxe de uma linguagem de programação. A ideia é motivar o aprendizado de conceitos de programação por meio de uma experiência divertida, envolvendo os alunos na elaboração de projetos como animações interativas, jogos digitais (MALONEY et al., 2010), entre outros recursos visuais.

De acordo com Zaharija et al. (2013), com o apoio dos recursos do Scratch, a criança ou jovem aprende a pensar e a trabalhar de forma criativa, sistemática e colaborativa. A interface gráfica do Scratch, e o recurso de "blocos de comandos" organizados dentro de diversas categorias como "movimento", "loops" etc., permitem que os programas sejam desenvolvidos sem a necessidade de memorização de linguagens e códigos de programação. A programação é feita a partir de fragmentos de código, embutidos nos blocos de comandos de diferentes cores e formatos, que podem ser arrastados para uma janela onde o programa é construído.

\section{Trabalhos relacionados}

$\mathrm{Na}$ linha do que se pretendeu desenvolver com o projeto aqui apresentado, encontramos outras iniciativas que buscam explorar os conceitos computacionais no contexto escolar de crianças e jovens. É o caso, por exemplo, do trabalho desenvolvido por França e Amaral (2013), que teve como objetivo estimular o desenvolvimento do "pensamento computacional" junto de alunos do ensino básico de uma escola pública, por meio do oferecimento de oficinas usando o Scratch. Os resultados do trabalho apontam que os alunos aprenderam determinados conceitos relacionados à área da computação e conseguiram aplicar o conhecimento adquirido para solucionar problemas.

Envolvendo alunos do ensino médio, o trabalho de Scaico et al. (2012) visava promover o desenvolvimento de habilidades de programação por meio de uma abordagem de ensino "orientada ao design". Assim, respeitando os princípios dessa abordagem, os alunos foram incentivados a elaborar algoritmos e a desenvolver seus projetos usando a criatividade e respeitando seus interesses pessoais. Com o intuito de promover a autonomia tecnológica dos jovens, no que diz respeito à apropriação dos recursos de programação para desenvolver o raciocínio lógico e resolver problemas, Arantes et al. (2014) propõem o desenho metodológico de um modelo de oficina extracurricular para explorar os recursos do Scratch. O projeto piloto envolveu nove alunos do ensino médio de uma escola pública em oito encontros e permitiu o levantamento das potencialidades e limites que um trabalho desse tipo pode enfrentar.

O ensino de programação para crianças do ensino fundamental foi proposto por Zaharija et al. (2013), como forma de diminuir, no futuro, o abandono dos cursos de programação. Os autores apresentaram uma revisão das principais ferramentas que podem facilitar o ensino e a aprendizagem de programação. Softwares como o Scratch, LEGO NXT-G e o RoboMind foram brevemente discutidos e suas possibilidades educacionais avaliadas. Com base nas limitações didáticas identificadas foi proposto um método de ensino de programação dividido em quatro fases. A proposta era que, na 
terceira fase, as crianças fizessem conexões lógicas entre a interação do robô no ambiente virtual e um "robô real". $\mathrm{Na}$ fase final as crianças programaram alguns movimentos em um "robô real" usando os recursos do LEGO NXT-G.

\section{Metodologia}

O trabalho aqui apresentado priorizou procedimentos metodológicos nos quais o uso da tecnologia auxiliou na construção e explicitação do conhecimento. Nesse caso, métodos que coloquem a tecnologia ao serviço do aprendiz (PAPERT, 1986), ou seja, que promovam o engajamento dos envolvidos no planejamento e elaboração de um "produto", que tenha um significado pessoal, e os levem a refletir sobre o processo de aprendizagem vivenciado (VALENTE, 2012).

Assim, foram utilizados os pressupostos relacionados à pedagogia de projetos, que possibilitam o "aprender-fazendo", e o reconhecimento da própria autoria no que foi produzido, por meio de questões que incentivam a contextualização dos conceitos aprendidos e a descoberta de outros conceitos que surgem durante o desenvolvimento do projeto (PRADO, 2003). Segundo Prado (2003), em situações de aprendizagem dessa natureza, o aprendiz acaba por desenvolver competências interpessoais, já que é necessário selecionar as informações relevantes, tomar decisões, trabalhar em grupo e administrar o confronto de ideias para aprender de forma colaborativa com seus pares.

À luz da metodologia definida, e sob o tema "Pensamento Computacional: transformando ideias em programas de computador usando Scratch", a proposta apresentada ao programa de Pré-IC para os anos letivos de 2014 (segundo semestre) e 2015 (primeiro semestre), previa atividades presenciais que estimulassem os alunos participantes a trocarem ideias e implementá-las usando programas de computador interativos e simples, no caso o Scratch. Pretendia-se, assim, oferecer os subsídios necessários para que os alunos se apropriassem de conhecimentos de computação e de inovação tecnológica que poderiam ser úteis, também, para motivá-los a seguir uma carreira na área das ciências exatas.

Ao todo, sete alunos e uma professora do $1^{\circ}$ ano do Ensino Médio de uma escola pública do interior do Estado de São Paulo, além de professores, alunos de pósgraduação e pesquisadores do Laboratório de Computação Aplicada à Educação (CAEd) participaram do projeto $^{3}$. Para traçar o perfil dos alunos participantes foi aplicado um questionário que investigou especialmente quais tecnologias os alunos têm disponíveis, e quais usos fazem delas em suas atividades diárias. Também foram inquiridos sobre o contato com linguagens de programação e suas expectativas em relação à participação no programa de Pré-IC. Depois disso, o plano de atividades previsto para ser desenvolvido em três momentos: (i) exploração dos recursos do Scratch; ii) planejamento, implementação e avaliação de um "projeto" de jogo educativo; iii) elaboração do relatório final de atividades), foi discutido e apresentado para aprovação de todos. Os doze meses estabelecidos para desenvolvimento do projeto foram distribuídos entre Maio de 2014 e Abril de 2015. A Figura 1 exibe as atividades desenvolvidas nesse período.

\footnotetext{
${ }^{3}$ Os autores agradecem a participação ativa de todos os alunos e pesquisadores do CAEd e aos professores e alunos da Escola Álvaro Guião, de São Carlos-SP, pelo envolvimento no projeto.
} 


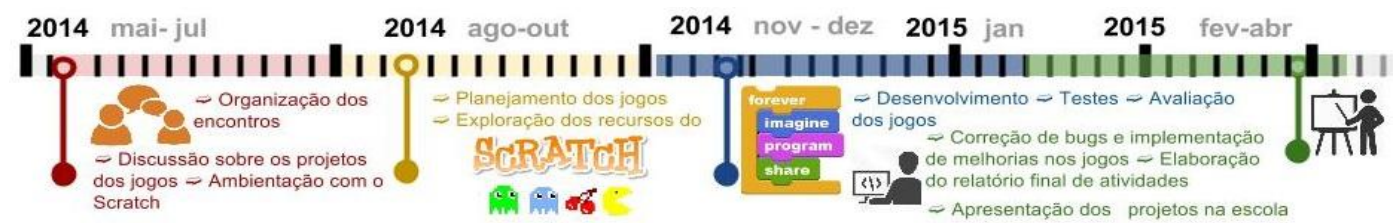

Figura 1 - Linha do tempo das atividades do Pré-IC 2014-2015

No total, foram cinquenta encontros, sendo dois encontros semanais, de 2 horas cada, realizados nos laboratórios de computação da Universidade.

\section{Perfil dos alunos participantes}

Dos 7 alunos participantes, com idade entre 14 e 16 anos, a maioria (6) é do sexo feminino, sendo que apenas 1 aluno participante é do sexo masculino. Todos os alunos entrevistados utilizam tecnologias no seu dia a dia, sendo que a maioria afirma que aprendeu a usar o computador sozinho (5 alunos) e o restante ( 2 alunos) com seus familiares. Para este grupo, a escola, os professores e os amigos não tem participação nessa aprendizagem. Nenhum aluno possui formação técnica em informática ou similar.

Sobre a utilização das tecnologias digitais no dia-a-dia foi possível verificar a preferência dos alunos entrevistados por equipamentos que permitem a mobilidade e a conectividade (smartphone e tablet), em detrimento de outras tecnologias digitais que não possuem tais características (computador desktop e câmera fotográfica). Em relação ao tipo de tecnologia e ao tempo dedicado à sua utilização diária, a maioria dos alunos entrevistados (6 alunos) dedicam em média 6 horas ou mais no uso do aparelho celular (smartphone). Apenas uma aluna não faz uso do recurso porque não o possui.

Quanto à prática de atividades diárias com tecnologias, a comunicação online é a atividade que os alunos entrevistados mais dedicam o seu tempo. Tanto a comunicação com colegas por mensagens digitais instantâneas quanto por meio da participação nas redes sociais, reforçam a preferência pelos equipamentos que permitem uma maior conexão e mobilidade. Em termos de produção de conteúdos, a realização e edição de fotos é a atividade mais desenvolvida pelo grupo. Participar de cursos online, desenhar, escrever em blogs, programar e editar filmes, são atividades pouco ou nada praticadas. Especificamente sobre o uso de linguagens de programação, apenas uma aluna afirma ter usado uma linguagem de programação, mencionando o $H T M L$, utilizado para editar seus posts no Tumblr. Quanto ao que os motivou a participar do projeto, a maioria afirma ter muito interesse ( 5 alunos) ou algum interesse ( 2 alunos) em aprender mais sobre as tecnologias de um modo geral e conhecer melhor o ambiente da universidade. Desenvolver programas para computador despertou muito interesse de 4 dos 7 alunos e o interesse de 3 alunos do grupo. Se divertir aprendendo coisas novas foi o que despertou o interesse de 6 alunos e muito interesse de 1 dos participantes. Melhorar o desempenho na escola aparece como interessante para a maioria, sendo 4 os alunos que afirmam ter interesse e outros 3 alunos algum interesse nesse aspecto do projeto.

\section{Desenvolvimento das atividades}

Conforme mencionado, o desenvolvimento das atividades planejadas compreendeu especificamente três momentos: i) exploração dos recursos do Scratch; ii) planejamento, implementação e avaliação de um "projeto" de jogo educativo; iii) elaboração do relatório final de atividades. 
O momento de exploração dos recursos do Scratch envolveu atividades de apresentação e discussão sobre os conceitos de lógica de programação e de aplicação prática desses conceitos usando os recursos do Scratch. Para isso, em cada encontro era proposto um desafio lúdico onde os alunos tinham que resolver, individualmente, determinada situação hipotética usando os componentes do aplicativo. Por exemplo, foi pedido aos alunos para criar um cenário com um personagem exposto às condições climáticas. Toda vez que começasse a chover o personagem deveria recorrer a um guarda-chuva para se proteger. Nesse cenário foi trabalhada a lógica do "Se... então", usando a estrutura if... then por meio da manipulação dos blocos de comandos condicionais disponíveis na interface do Scratch.

Para planejar, implementar e avaliar um "projeto" de criação de um jogo digital educativo, foi sugerido que os alunos trabalhassem em grupos para que, além de tomar conhecimento das etapas e procedimentos adotados no processo de desenvolvimento do projeto em si, também pudessem vivenciar uma situação de aprendizagem colaborativa e desenvolvessem habilidades interpessoais. Foram formadas, por afinidade, duas duplas e um trio. No início, as atividades de planejamento do projeto eram intercaladas com as atividades de discussão da lógica e exploração dos recursos do Scratch. A partir do sexto mês as atividades mais gerais, de definição de conceitos e exploração dos recursos do Scratch, estiveram voltadas à implementação de rotinas específicas definidas nos projetos propostos por cada grupo. Para auxiliá-los no planejamento do projeto foi compartilhado, em um editor de texto colaborativo ${ }^{4}$, um documento com algumas questões norteadoras, para ser preenchido com os requisitos de cada proposta, bem como para organização e acompanhamento do cronograma de trabalho. As propostas elaboradas geraram três jogos educativos ${ }^{5}$ : Operação IV; TreiENEM $e$ R3cicl@!.

O projeto Operação $I V$ foi pensado para ser um jogo educativo de matemática. O jogo apresenta as quatro operações matemáticas (adição, subtração, divisão e multiplicação) sugerindo contas aleatórias para que o jogador as resolva calculando. A Figura 2 apresenta a tela com as quatro operações tratadas no jogo, em formato de uma roleta, e alguns dos blocos de comandos usados pelos alunos para compor a rotina principal do jogo.

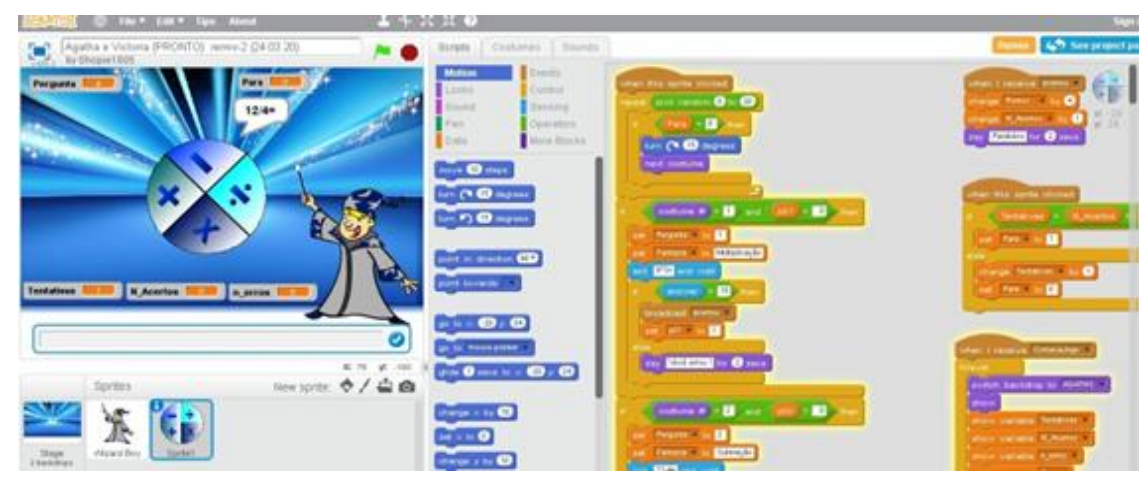

Figura 2 - Tela do jogo Operação IV e exemplo de alguns blocos de comandos usados

\footnotetext{
${ }^{4}$ O GoogleDocs (ou Documentos do GoogleDrive) é um editor de textos online que permite aos usuários criar e editar documentos de forma colaborativa, em tempo real.https://docs.google.com/document/

${ }^{5}$ Os jogos desenvolvidos estão disponíveis em https://scratch.mit.edu/studios/501942/
} 
Ao clicar na roleta, uma das operações é selecionada pelo personagem e as contas são exibidas para o jogador inserir a resposta. A cada resposta uma mensagem de acerto ou erro é apresentada e, caso esteja correta, a pontuação do jogador é incrementada.

Já o projeto TreiENEM é um jogo de perguntas e respostas sobre conceitos de matemática, voltado para quem quer aprimorar seus conhecimentos e se preparar para as provas dos vestibulares. As questões foram extraídas do Exame Nacional do Ensino Médio (ENEM) e são exibidas aleatoriamente na tela a partir de um comando do jogador. A Figura 3 mostra a tela padrão do jogo com uma das questões do ENEM e, no detalhe, um trecho dos blocos de comandos usados na confecção do jogo.

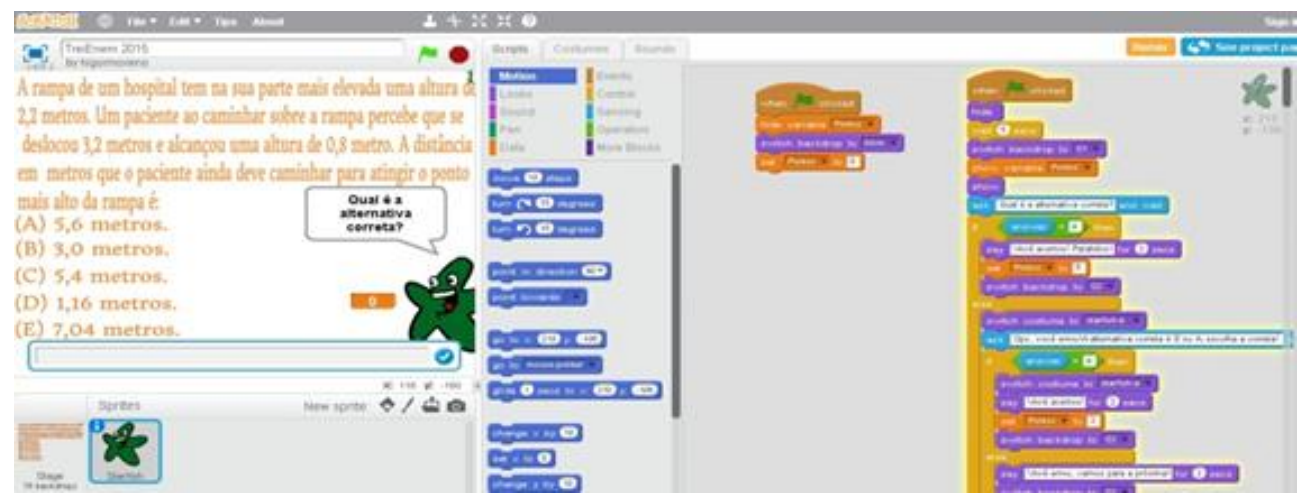

Figura 3 - Tela do jogo TreiENEM e exemplo de alguns blocos de comandos usados

Por sua vez, o projeto R3cicl@! busca conscientizar os jogadores com relação aos cuidados com o Meio Ambiente, especialmente no que diz respeito à compreensão de que é necessário reciclar o lixo não orgânico. Composto por cinco cenários distintos, a proposta do jogo é fazer com que o personagem recolha os materiais recicláveis (papel, metal, plástico e vidro) e os deposite nas lixeiras adequadas. A Figura 4, exibe a tela de um dos cenários e alguns blocos de comando usados para implementar o jogo.

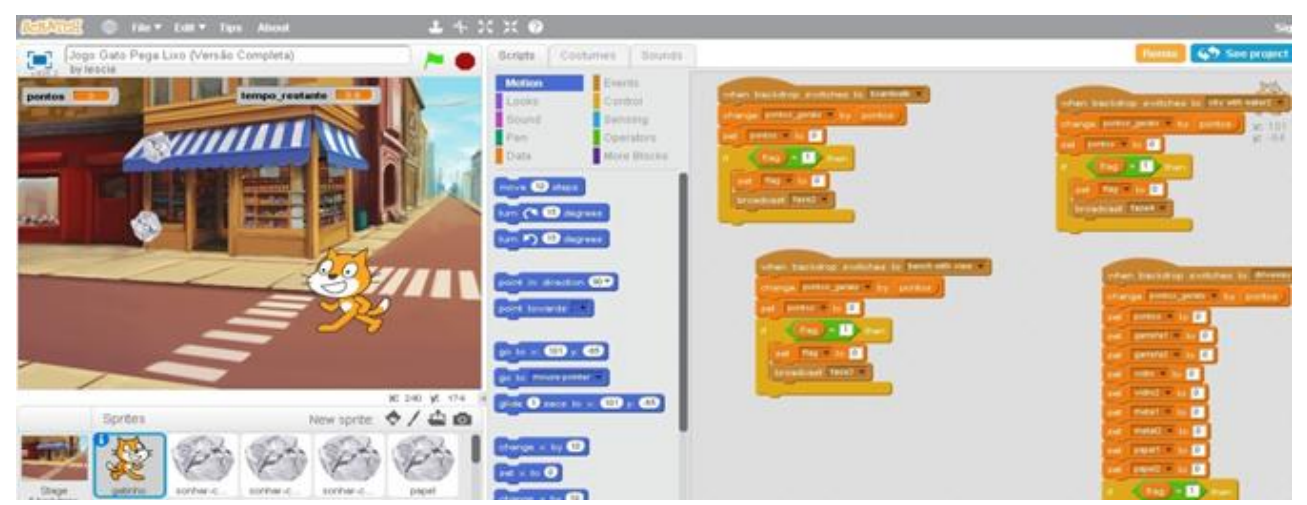

Figura 4 - Tela do jogo R3cicl@e exemplo de alguns blocos de comandos usados

Apesar de trabalharem os conceitos e a lógica de programação sob monitoria dos integrantes do CAEd, os alunos tiveram a liberdade de debater entre eles e implementar o seu próprio raciocínio, o que gerou interessantes e diferentes resultados.

O relatório final de atividades é um item obrigatório, redigido individualmente pelo aluno participante, que deve conter as atividades desenvolvidas durante o período de participação no projeto e uma reflexão crítica sobre o seu envolvimento nas atividades propostas. O momento de registro e reflexão sobre as atividades 
desenvolvidas não foi uma tarefa fácil de ser realizada pelos alunos, que tiveram dificuldade em explicitar, por escrito, a experiência vivida no projeto.

Além das atividades desenvolvidas nos laboratórios do ICMC, os alunos também apresentaram seus projetos na universidade e para a comunidade escolar, assim como participaram de eventos ocorridos na Universidade, entre eles o SEMComp 2014, tendo oportunidade de vivenciar de outra forma o ambiente acadêmico.

\section{Resultados alcançados}

O projeto aqui apresentado tinha como principal objetivo desenvolver noções básicas do "pensamento computacional" junto de sete alunos do primeiro ano do Ensino Médio de uma escola pública, por meio dos recursos do Scratch.

Por ser uma proposta realizada no âmbito de um programa de Pré-IC, também era necessário proporcionar aos alunos participantes uma vivência acadêmica, especialmente no que diz respeito à pesquisa científica. Assim, por meio dos pressupostos metodológicos relacionados à pedagogia de projetos, foi possível conduzir atividades teórico-práticas que favoreceram a descoberta e a materialização de conceitos relacionados ao "pensamento computacional", bem como o desenvolvimento de competências interpessoais e de pesquisa.

Como resultado direto dos procedimentos adotados nas atividades de exploração dos recursos do Scratch, destaca-se a possibilidade de estimular o raciocínio lógico e a resolução de problemas de forma lúdica e dinâmica, além do desenvolvimento de noções básicas de programação. Já nos momentos de planejamento, implementação e avaliação do "projeto" de jogo educativo, as atividades propostas possibilitaram a implementação de três "produtos" concretos que permitiram verificar, na prática, como os alunos participantes desenvolveram habilidades de seleção e organização de informações e se apropriaram dos recursos cognitivos inerentes ao "pensamento computacional". As atividades de implementação dos jogos digitais, por serem de natureza colaborativa, possibilitaram ainda estimular a resolução de conflitos e a tomada de decisão para atingirem um objetivo comum.

O relatório final das atividades resultou em um exercício importante de reflexão e síntese e, apesar das dificuldades encontradas pelos alunos para registrarem suas impressões, acabou por ser um instrumento de autoavaliação e confirmação dos resultados alcançados pelo engajamento no projeto. Depoimentos como, por exemplo, o de uma aluna que registrou a descoberta da "dinâmica" por trás de um jogo de computador: "Aprendi a pensar em como as coisas funcionam, olhar sua engenharia e também a desenvolvê-las. Jogando outros jogos, comecei a analisá-los, e tentar entender como foram programados, quais os comandos, quais variáveis utilizadas, entre outras coisas... (isso) me ajudou a ser mais crítica, e a pensar além da aparência de um jogo/programa", confirma que o objetivo foi atingido. Outro resultado importante foi mostrar aos alunos participantes que a Universidade não é uma realidade distante e que é possível ter acesso a ela, a partir da dedicação e comprometimento com os estudos. Esse resultado pôde ser comprovado nos relatórios de todos os alunos, em registros como, por exemplo: "Só por poder estar dentro de uma universidade, em minha própria cidade, isto se torna mais gratificante ainda e sei que, ter vivenciado essa experiência, irá me ajudar no meu percurso acadêmico". 
Outro resultado indireto relevante derivou do fato dos alunos apresentarem os jogos criados por eles na escola em que estudam. Essa ação contribuiu para aproximar a universidade da comunidade local, sensibilizando os docentes e demais alunos a participarem de novos projetos no futuro.

\section{Considerações finais}

Apesar dos resultados terem sido positivos, indicando que os objetivos foram alcançados, é importante fazer algumas considerações sobre as limitações e possibilidades de ampliação do trabalho apresentado.

Em relação ao uso do Scratch para a implementação dos jogos digitais, verificou-se que este apresenta algumas limitações quanto à aplicação em projetos de grupo, uma vez que seus recursos não permitem o trabalho colaborativo. Esse fato impossibilitou aos alunos compartilharem uma única área de trabalho e fez com que os grupos tivessem que optar por desenvolver o projeto na conta Scratch de um único aluno, gerando algum desconforto quanto à divisão de tarefas e para o acesso e continuidade do trabalho quando o responsável pela conta não estava presente.

No que diz respeito ao planejamento dos projetos dos jogos, talvez seja interessante orientar os alunos sobre as características dos diferentes tipos de jogos que podem ser implementados usando os recursos do Scratch. A pouca orientação nesse sentido fez com que um dos projetos explorasse características mais típicas de um jogo de perguntas e respostas (TreiENEM), não investindo nas possibilidades mais interativas permitidas pelo aplicativo como, por exemplo, rotinas necessárias para a movimentação de personagens e objetos, que surgiram nos outros dois jogos criados (Operação IV e R3cicl@)).

Por fim, é importante salientar que iniciativas de Pré-IC, apesar da limitação em relação à quantidade de alunos que podem ser atendidos, são importantes para despertar o interesse por seguir uma carreira universitária e fomentar um maior envolvimento da comunidade com as universidades de sua região. No caso desse trabalho, o programa de Pré-IC criou oportunidade para que os sete alunos participantes pudessem complementar sua formação pessoal, contribuindo para o aprimoramento de seus conhecimentos além de auxiliá-los na preparação para a vida acadêmica e profissional.

\section{Agradecimentos}

Os autores agradecem à FAPESP, CAPES e ao CNPq pelo apoio.

\section{Referências}

Arantes, F. L.; Amiel, T.; Fedel, G. (2014) "Nos rumos da autonomia tecnológica desafios e lições aprendidas para a formação de jovens". $3^{\circ}$ Congresso Brasileiro de Informática na Educação (CBIE 2014) 20a Workshop de Informática na Escola (WIE 2014), pp. 308-317.

Costa, F. A.; Rodriguez, C.L.; Cruz, E.; Fradão, S. (2012). "Repensar as TIC na educação: o professor como agente transformador. 1 ed. Lisboa: Santillana.

Easterbrook, S. (2014) "From Computational Thinking to Systems Thinking: A conceptual toolkit for sustainability computing". Proceedings of the 2nd International Conference on Information and Communication Technologies for 
CBIE-LACLO 2015

Anais do XXI Workshop de Informática na Escola (WIE 2015)

Sustainability (ICT4S'2014), Stockholm, Sweden, 24-27 August, 2014.

França, R. S. de; Amaral, H. J. C. do. (2013) "Proposta Metodológica de Ensino e Avaliação para o Desenvolvimento do Pensamento Computacional com o Uso do Scratch". II Congresso Brasileiro de Informática na Educação (CBIE 2013) XIX Workshop de Informática na Escola (WIE 2013), pp. 179-188.

Gatti, B. A. (2000) "Formação de professores e carreira: problemas e movimentos de renovação". 2.ed. Campinas: Autores Associados.

Lee, I. (2014) "CSTA Computational Thinking Task Force".

Maloney, J.; Resnick, M.; Rusk, N. ; Silverman, B.; Eastmond, E. (2010) "The scratch programming language and environment". ACM Transactions on Computing Education (TOCE), 10 (4), 16.

MCT (2000). Ministério da Ciência e Tecnologia. Sociedade da Informação no Brasil. Livro Verde. Tadao Takahashi (org.). Brasília, Setembro, 2000. Disponível em: <http://livroaberto.ibict.br/bitstream/1/434/1/Livro\%20Verde.pdf>. Acesso em 14 Maio 2015.

Nunes, D. J. (2011) "Ciência da Computação na Educação Básica”. Jornal da Ciência. 09de setembro, 2011..

Papert, S. (1986) "Constructionism: a new opportunity for elementary science education". Massachusetts Institute of Technology.The Epistemology and Learning Group. Proposta para a National Science Foundation..

Prado, M. E. B. (2003) "Pedagogia de Projetos. Série "Pedagogia de Projetos e Integração de Mídias" - Programa Salto para o Futuro, Setembro, 2003. Disponível em: <http://www.eadconsultoria.com.br/matapoio/biblioteca/textos_pdf/texo18.pdf $>$. Acesso em 04 Maio 2015.

Scaico, P. D.; Lima, A. A. de; Silva, J. B. da; Azevedo, S.; Paiva, L. F.; Raposo, E. H. S.; Alencar, Y.; Mendes, J. P. (2012) "Programação no Ensino Médio: Uma Abordagem de Ensino Orientado ao Design com Scratch”. Anais do XVIII WIE Rio de Janeiro, 26 a 30 de Novembro de 2012, pp.

Valente, J. A. (2002) "A espiral da aprendizagem e as tecnologias da informação e comunicação: repensando conceitos". In: JOLY, M.C.R.A. (Ed.). A tecnologia no ensino: implicações para a aprendizagem. São Paulo: Casa do Psicólogo Editora.

Valente, J. A. (2003) "Pesquisa, comunicação e aprendizagem com o computador". Série "Pedagogia de Projetos e Integração de Mídias" - Programa Salto para o Futuro, Setembro, 2003.

Zaharija, G.; Mladenovic, S.; Boljat, I. (2013) "Introducing basic Programming Concepts to Elementary School Children", Procedia - Social and Behavioral Sciences, vol. 106, pp. 1576-1584.

Wing, J. (2006) “Computational Thinking”. Communications of the ACM, vol. 49, no. 3, pp. 33-35. 